\title{
Short-Latency Visual Input to the Subthalamic Nucleus Is Provided by the Midbrain Superior Colliculus
}

\author{
Véronique Coizet, ${ }^{1,2}$ John H. Graham, ${ }^{3}$ Jonathan Moss, ${ }^{4}$ J. Paul Bolam, ${ }^{4}$ Marc Savasta, ${ }^{2}$ John G. McHaffie, ${ }^{5}$ \\ Peter Redgrave, ${ }^{1}$ and Paul G. Overton ${ }^{1}$ \\ ${ }^{1}$ Department of Psychology, University of Sheffield, Sheffield S10 2TP, United Kingdom, ${ }^{2}$ Inserm U836, Grenoble Institute of Neurosciences, Joseph Fourier \\ University, Grenoble 38042, France, ${ }^{3}$ Department of Biology, King College, Bristol, Tennessee 37620, ${ }^{4}$ Medical Research Council Anatomical \\ Neuropharmacology Unit, University of Oxford, Oxford OX1 3TH, United Kingdom, and ${ }^{5}$ Department of Neurobiology and Anatomy, Wake Forest \\ University School of Medicine, Winston-Salem, North Carolina 27157
}

The subthalamic nucleus (STN) is one of the principal input nuclei of the basal ganglia. Using electrophysiological techniques in anesthetized rats, we show that the STN becomes responsive to visual stimuli at short latencies when local disinhibitory injections are made into the midbrain superior colliculus (SC), an important subcortical visual structure. Significantly, only injections into the lateral, but not medial, deep layers of the SC were effective. Corresponding disinhibition of primary visual cortex also was ineffective. Complementary anatomical analyses revealed a strong, regionally specific projection from the deep layers of the lateral SC to neurons in rostral and dorsal sectors of the STN. Given the retinocentric organization of the SC, these results suggest that lower-field stimuli represented in the lateral colliculus have a direct means of communicating with the basal ganglia via the STN that is not afforded to visual events occurring in the upper visual field.

\section{Introduction}

The subthalamic nucleus (STN) occupies a central position in the functional architecture of the basal ganglia (Parent and Hazrati, 1995; Joel and Weiner, 1997), an interconnected group of subcortical nuclei that represent one of the brain's fundamental processing units (Gerfen and Wilson, 1996; Redgrave, 2007). Despite a prodigious volume of work, there is no agreed consensus concerning the function(s) of the basal ganglia; however, their dysfunction is accompanied by a well characterized range of symptoms which underlie such disorders as Huntington's disease and Parkinson's disease (Wichmann and DeLong, 1996; Obeso et al., 2000). In the latter case, the STN has achieved particular prominence in recent years as a therapeutic target for deep brain stimulation (Benabid, 2003). Nevertheless, our knowledge of the STN, certainly compared with other basal ganglia nuclei, is still limited, and this deficiency seriously impedes our understanding of basal ganglia function and the development of interventions to ameliorate their dysfunction. In particular, although the interrelationship of the STN with other basal ganglia nuclei is well documented (Parent and Hazrati, 1995; Joel and Weiner, 1997), its regulation by external systems is not, even though it is increas-

Received Dec. 11, 2008; revised Feb. 27, 2009; accepted April 2, 2009.

This work was funded by grants from the Biotechnology and Biological Sciences Research Council (to P.G.O.: 062742), Wellcome Trust (to P.R.: WT080943), Medical Research Council, UK (to J.P.B. and J.M.), and National Institutes of Health (to J.G.M.: NS35008). We are grateful to Natalie Kennerley for her excellent histological assistance and to Peter Furness for the provision of software to analyze the electrophysiological data.

Correspondence should be addressed to Paul G. Overton, Department of Psychology, University of Sheffield, Western Bank, Sheffield, South Yorkshire S10 2TP, UK. E-mail: p.g.overton@sheffield.ac.uk.

DOI:10.1523/JNEUROSCI.0247-09.2009

Copyright (C) 2009 Society for Neuroscience $\quad 0270-6474 / 09 / 295701-09 \$ 15.00 / 0$ ingly considered to be a major entry point to the basal ganglia (Nambu et al., 2002).

Electrophysiological studies have revealed that the STN contains neurons whose activity is related to movement and others that respond to sensory stimuli (DeLong et al., 1985; Matsumura et al., 1992). This suggests the structure plays a role in transforming sensory signals into a behaviorally relevant output. The transformation(s) performed will, in part, be determined by the informational content of the sensory signals coded by STN neurons. This in turn will be constrained by the perceptual properties of afferent sensory circuits. One strategy therefore to reveal the content of sensory signals encoded by STN neurons would be to determine the perceptual properties of afferent sensory structures. However, the origin of sensory inputs to the STN has remained largely unexplored.

Neurons in the STN exhibit short-latency (70-120 ms) responses to the onset of phasic visual stimuli (Matsumura et al., 1992). Retinal projections to either the geniculostriate system or the midbrain superior colliculus (SC) are the most likely sources of short-latency visual activation. However, the visual cortex provides no direct input to the STN; indeed, cortical afferents arise exclusively from prefrontal and motor areas (Afsharpour, 1985b; Nambu et al., 2002). Recently, we have shown that short-latency visual input to another important input station of the basal ganglia, the dopaminergic neurons of the ventral midbrain, is provided by the SC (Comoli et al., 2003; Dommett et al., 2005), a highly conserved but evolutionarily ancient subcortical visual structure. This raises the intriguing possibility that the SC may also act as the primary source of short-latency visual input to the STN. Our present results confirm this supposition, first by establishing that the SC is critical for short-latency visual activation of 
lateral superior colliculus

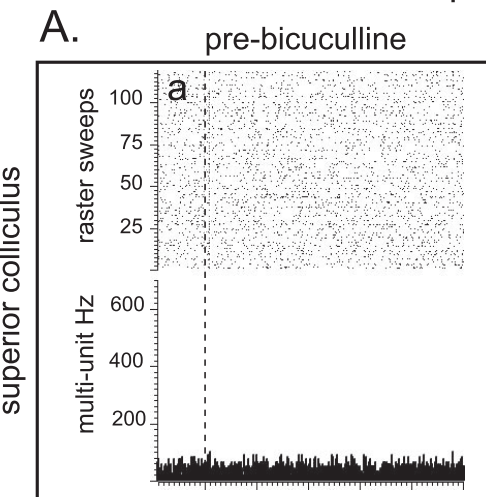
post-bicuculline
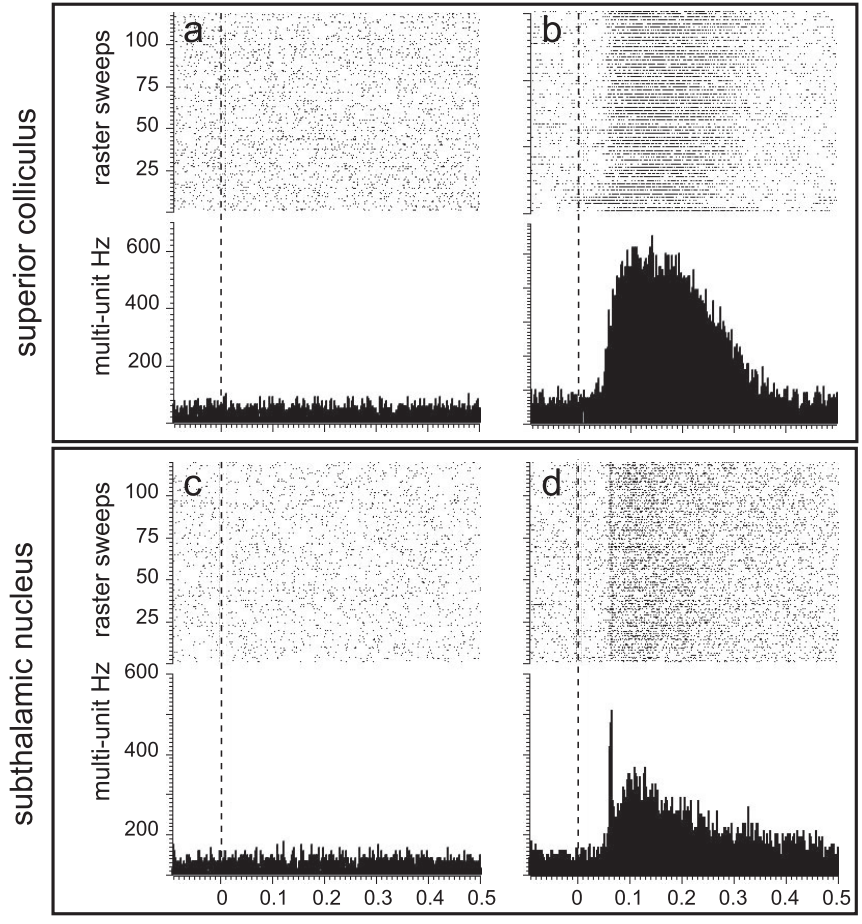

time (s) medial superior colliculus
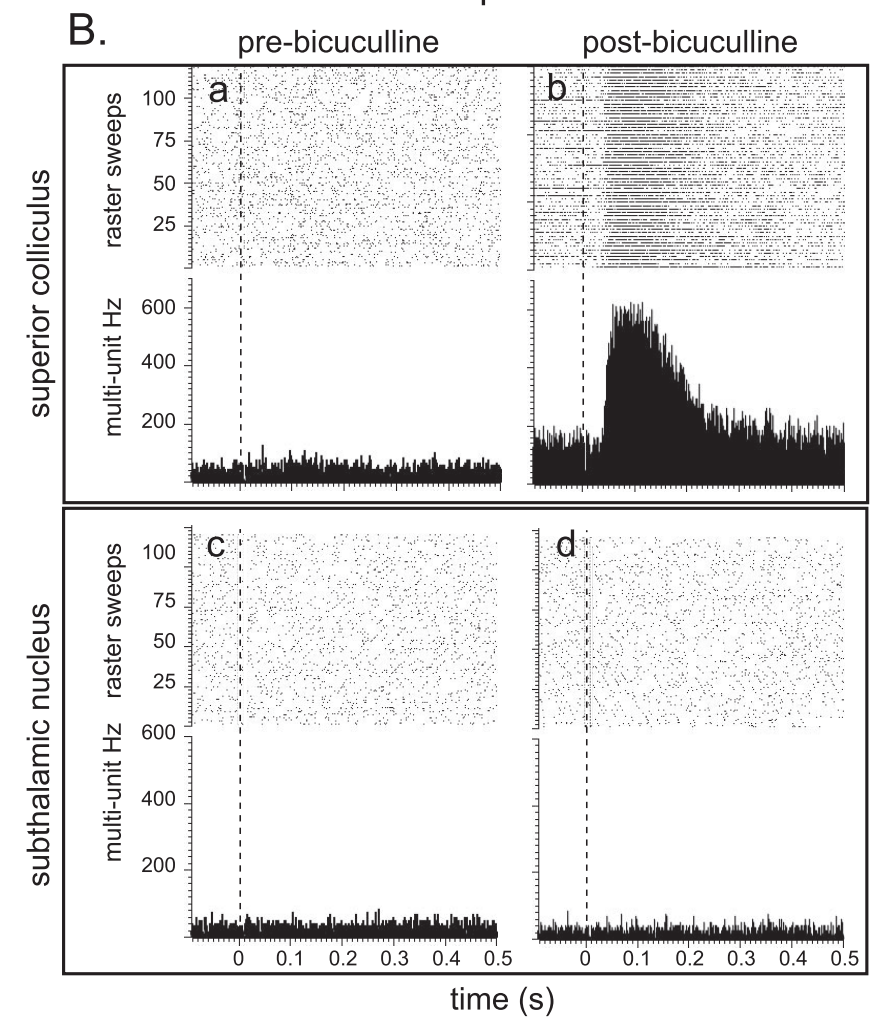

Figure 1. Effects of local intracollicular injections of bicuculline on visually evoked multiunit responses in the SC and STN. The graphs present raster displays and peristimulus histograms of single-case data aligned on the presentation of 120 light flashes $(0.5 \mathrm{~Hz})$ delivered to the contralateral eye (vertical dotted line). $A$ represents data from a case where bicuculline was injected into the lateral deep layers. $\boldsymbol{B}$ is from a case where bicuculline was injected into medial SC. In both cases, before the injection of bicuculline, neither the SC ( $\boldsymbol{A} \boldsymbol{a}, \boldsymbol{B} \boldsymbol{a})$ nor the $\mathrm{STN}(\boldsymbol{A c}, \boldsymbol{B} \boldsymbol{C})$ was responsive to the light flash. Following the injection of bicuculline into both lateral and medial SC, local neurons in the SC became responsive to the visual stimulus ( $\boldsymbol{A} \boldsymbol{b}$ and $\boldsymbol{B} \boldsymbol{b}$, respectively). In contrast, only when visual sensitivity was restored to the lateral SC did STN neurons become visually responsive (compare $\boldsymbol{A d}$ with $\boldsymbol{B d}$ ).

STN neurons and then by demonstrating the existence of a direct excitatory projection from a specific subregion of the SC to the STN.

\section{Materials and Methods}

All aspects of this study were performed with Home Office approval under section 5(4) of the Animals (Scientific Procedures) Act 1986, and experimental protocols received prior approval of the Institutional Ethics Committees.

\section{Electrophysiology}

Twenty-two male Hooded Lister rats (285-620 g) anesthetized with urethane (ethyl-carbamate, $1.25 \mathrm{~g} / \mathrm{kg}$, Sigma-Aldrich) were used in the electrophysiology experiments. A contralateral approach using an angle of $35^{\circ}$ (ML: 2.5-3.2, bregma) was used to position a parylene-C-insulated tungsten microelectrode (1-2 M $\Omega$, A-M Systems) into the STN (AP: 3.6-4.1 mm, bregma; DV: 7.0-8.0 mm, dura). A second tungsten electrode coupled with a 30 gauge metal injector needle filled with the $\mathrm{GABA}_{\mathrm{A}}$ receptor antagonist bicuculline methiodide (40 ng per $400 \mathrm{nl}$ of saline, Sigma-Aldrich) was introduced vertically into the SC (AP: 5.8$6.3 \mathrm{~mm}$, bregma; ML: $1.0-2.5 \mathrm{~mm}$, bregma; DV: $3.5-5.0 \mathrm{~mm}$, dura). Lateral separation between the electrode and the injector was $0.2-0.5$ $\mathrm{mm}$. Intracollicular injections of bicuculline $(0.5 \mu \mathrm{l} ; 0.5 \mu \mathrm{l} / \mathrm{min})$ were made with a $10 \mu \mathrm{l}$ Hamilton syringe (Scientific Laboratory Supplies) mounted on an infusion pump, connected to the needle by a length of plastic tubing. The experimental rationale in the present study was based on the important observation of Katsuta and Isa (2003) that local intracollicular injections of bicuculline can reinstate visual sensitivity to the SC deep layers rendered insensitive by urethane anesthesia. (Note that throughout the current article, we adhere to the convention of referring to all SC layers underlying the superficial layers as the "deep layers.")
Because of the known functional organization of the rodent SC (Dean et al., 1989), particular attention was paid to the effects of local disinhibition within different regions of the retinocentric map (Wurtz and $\mathrm{Al}-$ bano, 1980; Stein and Meredith, 1993).

Multiunit spike-related potentials from the STN and SC were amplified, bandpass filtered $(300 \mathrm{~Hz}$ to $10 \mathrm{kHz})$, digitized at $10 \mathrm{kHz}$, and recorded directly onto computer disc using a 1401+ hardware acquisition system (Cambridge Electronic Design) connected to a standard PC running CED Spike 2 software. Multiunit electrophysiological responses in the SC were used to guide the position of the SC electrode. Recordings were made while the electrode/injector assembly was lowered into the SC in the presence of a whole-field light flash $(0.5 \mathrm{~Hz}, 10 \mathrm{~ms}$ duration) from a green LED ( 60 lux $570 \mathrm{~nm}$ ) positioned $5 \mathrm{~mm}$ in front of the contralateral eye. Using the characteristically vigorous visual response of the SC superficial layers as a guide to position, the electrode was lowered into the subjacent intermediate layers of the medial or lateral sectors of the SC. When both the STN and SC electrodes were in place, sets of trials comprising 120 light flashes (parameters as above) were recorded. Responses were obtained during an initial baseline period before an SC injection of bicuculline. Sets of trials then were continued until the effect of the drug had worn off, typically $15-20 \mathrm{~min}$.

In three cases, an insulated silver wire was inserted into the visual cortex (with the dura carefully retracted) to record visually evoked field potentials. These were low-pass filtered (DC-1 kHz, $3 \mathrm{~dB}$ points), digitized at $10 \mathrm{kHz}$, and recorded directly to disk using the $1401+$ acquisition system. Signal averaging over sets of 300 light flashes was performed by the Spike 2 software. After recording under baseline conditions, a Gelfoam swab soaked in a solution of bicuculline $(100 \mu \mathrm{g} / \mathrm{ml})$ was placed directly on the surface of the exposed cortex. Averaged field potentials were recorded for successive series of 300 flashes until the effect of the drug had worn off. 

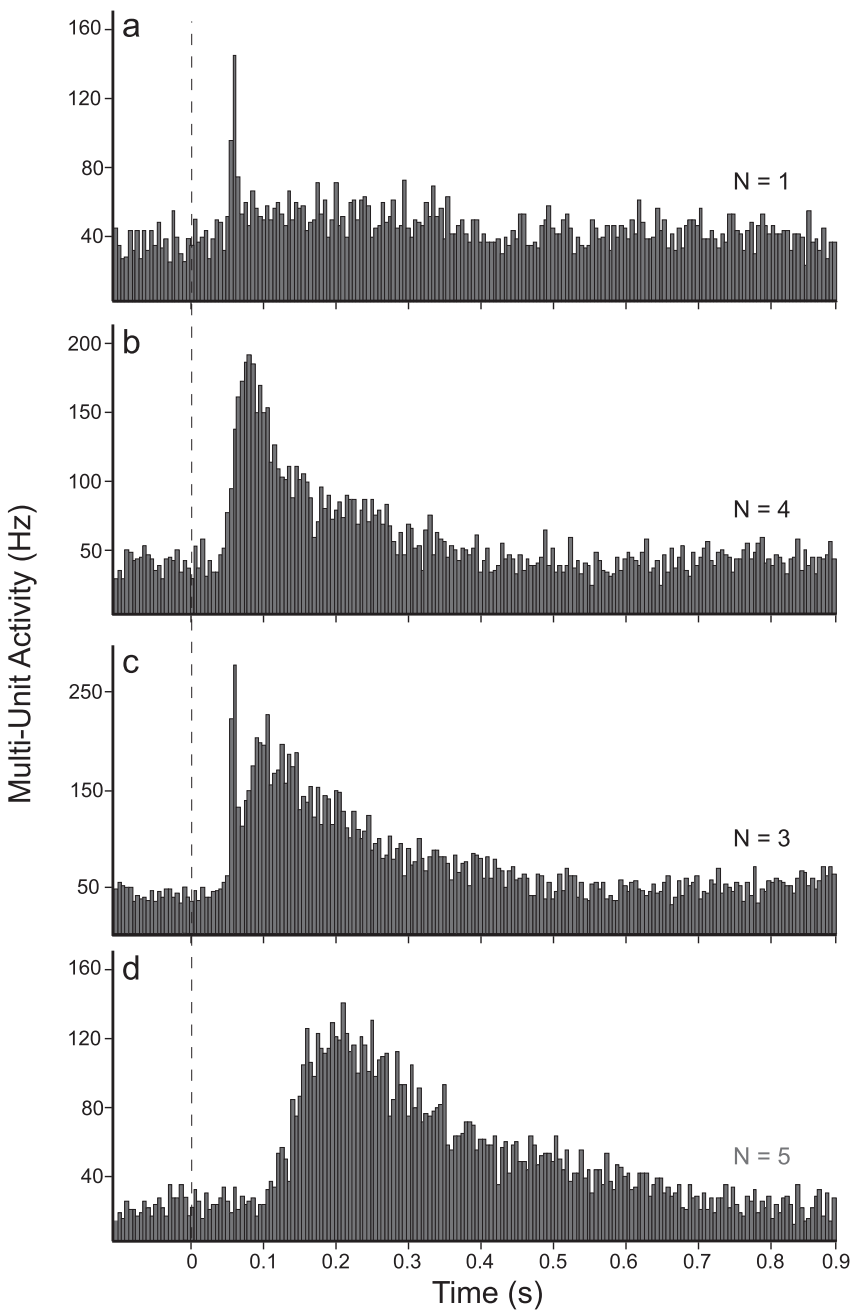

Figure 2. Peristimulus histograms showing individual cases of different types of shortlatency visually evoked responses in the STN. The dashed vertical line indicates the onset time of the visual stimulus. The $n$ associated with each histogram indicates the number of cases exhibiting that class of response; total $n=13$. $\boldsymbol{a}$, Short-latency ( $<70 \mathrm{~ms})$, very-short-duration $(10-15 \mathrm{~ms})$ response. $\boldsymbol{b}$, Short-latency $(<70 \mathrm{~ms})$, but slower-onset and longer-duration $(\sim 400 \mathrm{~ms}$ ) response. $\boldsymbol{c}$, A combination of response classes $\boldsymbol{a}$ and $\boldsymbol{b} ; \boldsymbol{d}$, A longer-duration (700$800 \mathrm{~ms}$ ) response with latency $>100 \mathrm{~ms}$.

At the end of the experiments, the position of any subcortical recording sites was marked with a small lesion induced by passing a $10 \mu \mathrm{A}$ anodal current for $2.5 \mathrm{~min}$ through the tungsten recording electrode. Animals then were killed by an overdose of pentobarbitone and perfused with $0.9 \%$ saline followed by $4 \%$ paraformaldehyde. The brains were taken for histological analysis.

Following the experiments, peristimulus time interval histograms (PSTHs) were constructed based on SC and STN multiunit data (bin width $1 \mathrm{~ms}$ ). PSTHs were imported into an Excel program (Microsoft) (Peter Furness, Sheffield) which determined the following response characteristics before and after an injection of bicuculline: (1) baseline activity: the mean number of multiunit spikes occurring during the $5001 \mathrm{~ms}$ bins before the light flash; (2) response latency: the time point at which poststimulation spikes exceeded 3.0 SD of the baseline mean; (3) response duration: response offset was defined as the time point at which poststimulation activity returned to $3.0 \mathrm{SD}$ of the baseline mean; and (4) response amplitude: the mean number of multiunit spikes between response onset and offset minus the baseline mean. The statistical reliability of differences between response latencies for the SC and STN, and between STN baseline firing rates before and after SC injections of bicuculline, were assessed by $t$ tests. Probabilities $<0.05$ (two tailed) were accepted as statistically reliable.

\section{Anatomy}

Tracer injections. For the light microscopy experiments, we used 15 male Hooded Lister rats (265-602 g) bred in the Sheffield laboratory. Animals were anesthetized with an intraperitoneal injection of a mixture of Ketaset $(0.765 \mathrm{ml} / \mathrm{kg})$ and Rompun $(1.1 \mathrm{ml} / \mathrm{kg})$. Single injections of the anterograde tracers biotinylated dextran amine (BDA: Sigma-Aldrich) and Phaseolus vulgaris leucoagglutinin (PHA-L: Vector Laboratories) were directed to different quadrants of the SC on opposite sides of the brain in the same subject (e.g., caudomedial and rostrolateral or caudolateral and rostromedial). The stereotaxic coordinates were as follows: AP: 6.0-7.3 $\mathrm{mm}$, bregma; ML: 0.5-2.5 mm, bregma; DV: $3.5-5.0 \mathrm{~mm}$, dura). Some injections of anterograde tracers were combined with an injection of the retrograde tracer Fluorogold (Fluorochrome) into the substantia nigra pars reticulata (AP: 5.0-5.8 mm, bregma; ML: 1.0-3.0 mm, bregma; DV: $8.0-9.0 \mathrm{~mm}$, dura) to help identify the STN. BDA (10\% in phosphate buffer; $\mathrm{PB})$ and Fluorogold (4.0\% in distilled water) were pressure ejected in volumes of 30-90 nl, while PHA-L was ejected iontophoretically $(5 \mu \mathrm{A}$ anodal current applied to a $2.5 \%$ solution in $\mathrm{PB}, 7 \mathrm{~s}$ on/off for 15-20 min). To identify SC neurons projecting to the STN, small $(6-10 \mathrm{nl})$ pressure ejections of the retrograde tracer cholera toxin subunit B (CTb; Quadratech Epsom; 1\% solution in $\mathrm{PB}$ ) or larger injections of the fluorescent tracer True Blue (Sigma-Aldrich) (5\% in distilled water) were made into the STN. After allowing $7 \mathrm{~d}$ for the transport of tracers, animals were reanesthetized with pentobarbitone and perfused transcardially with warm saline $\left(40^{\circ} \mathrm{C}\right)$ followed by $4 \%$ paraformaldehyde in $\mathrm{PB}, \mathrm{pH}$ 7.4. Serial coronal or sagittal sections $(30 \mu \mathrm{m})$ were cut on a freezing microtome and collected in PB, except for tissue containing True Blue, where the sections were collected directly onto slides, allowed to dry in a light protected box, and coverslipped with DPX mountant.

For electron microscopy, an additional three rats received PHA-L injections as described above. Following $7 \mathrm{~d}$ survival, the animals were perfused with PBS ( $0.01 \mathrm{M}$ phosphate) followed by $3 \%$ paraformaldehyde plus $0.1 \%$ glutaraldehyde. Sagittal sections $(70 \mu \mathrm{m})$ were cut on a vibratome and collected in PB.

Histology for light microscopy. To reveal the tracers (BDA, PHA-L, CTb, or Fluorogold), free-floating sections were washed with $0.1 \mathrm{M} \mathrm{PB}$ followed by 0.1 м PB containing $0.3 \%$ Triton X-100 (PB-TX) for $30 \mathrm{~min}$. For animals injected with PHA-L, CTb, or Fluorogold, the sections were incubated overnight in primary antibody solution (goat anti-PHA-L, 1:800-1,000 dilution, Vector Laboratories, or goat anti-CTb, 1:4000 dilution, Quadratech, or rabbit anti-Fluorogold, 1:50,000 dilution, Fluorochrome). The next day, sections were washed with PB-TX and incubated for $2 \mathrm{~h}$ in biotinylated rabbit anti-goat $\operatorname{IgG}(1: 100$, Vector Laboratories, in PB-TX containing 2\% normal rabbit serum) for PHA-L and CTb or in biotinylated goat anti-rabbit IgG (1:100, Vector Laboratories, in PB-TX containing $2 \%$ normal goat serum) for Fluorogold. After 30 min washing, all the sections were incubated in Elite Vectastain $\mathrm{ABC}$ reagent (Vector Laboratories, 1:100 in PB-TX) for $2 \mathrm{~h}$. The peroxidase associated with the tracers was revealed by reacting tissue with $\mathrm{H}_{2} \mathrm{O}_{2}$ for $\sim 1$ min using nickel-enhanced diaminobenzidine (DAB) as the chromogen for BDA and CTb (black reaction product) while PHA-L and Fluorogold were revealed by incubation with VIP (Vector Laboratories) chromogen (purple reaction product). Finally, sections were washed in $\mathrm{PB}$, mounted on gelatin-coated slides, dehydrated in graded dilutions of alcohol, cleared in xylene, and coverslipped in DPX.

Light microscopy, data analysis. Following injections of anterograde tracers into the SC, three coronal sections through the STN approximately equivalent to $3.6,3.8$, and $4.1 \mathrm{~mm}$ caudal to bregma in the atlas of Paxinos and Watson (1997), or three sagittal sections approximately equivalent to $1.9,2.4$, and $2.9 \mathrm{~mm}$ lateral to midline were selected for analysis. Photomicrographs of injection sites in the SC and of the STN on each section were taken with a Spot-2 digital camera mounted on a Nikon Eclipse 800.

The location of retrogradely labeled neurons was plotted on four coronal sections through the SC separated by $\sim 0.5 \mathrm{~mm}$ (equivalent to $5.8,6.3$, 6.8 , and $7.3 \mathrm{~mm}$ caudal to bregma). A series of digital images (magnification $100 \times$ ) were taken and imported into a graphics program (Macromedia Freehand, Adobe) where they were montaged. The borders and 
layers of the SC were drawn over the montage. The scheme used for identifying the layers of the rodent SC is the one we have used previously (Sahibzada et al., 1986; Dean et al., 1989; Redgrave et al., 1992) and is based on the morphometric study of Albers et al. (1988) (i.e., SGS, stratum griseum superficiale; SO, stratum opticum; SGI, stratum griseum intermediale; SAI, stratum album intermediale; SP, strata profunda). Each layer was bisected into medial and lateral zones as described previously (Telford et al., 1996). For each section, retrogradely labeled neurons appearing in each subdivision were plotted using a combination of the underlying photomontage and direct microscopic inspection of the tissue. A three-factor repeatedmeasures ANOVA was used to analyze differences in the distribution of tectosubthalamic neurons within different sectors of the SC. The factors were as follows: (1) "rostral/ caudal" with four levels $(5.8,6.3,6.8$, and 7.3$)$; (2) "medial/lateral" with two levels (medial or lateral); and (3) "layers" with four levels (SGS+SO, SGI, SAI, and SP). Probabilities $<0.05$ (two tailed) were accepted as statistically reliable.

Electron microscopy, histology and analysis. Tissue for electron microscopic analysis was treated in a similar manner, except that the sections were freeze thawed using isopentane and liquid nitrogen, and Triton X-100 was not used. PHA-L was revealed using DAB as the chromogen for the peroxidase reactions $(\sim 6 \mathrm{~min})$. After postfixation in osmium tetroxide ( $1 \%$ in $\mathrm{PB}$, $30 \mathrm{~min}$ ), sections were embedded in resin and flat mounted on microscope slides. Regions of the STN with anterograde labeling were reembedded and resectioned for electron microscopic analysis. The sections were collected on Pioloform-coated, single-slot grids that were stained with lead citrate and examined in a Philips CM10 electron microscope.

\section{Results}

\section{Electrophysiology}

Visually evoked activation of the SC and STN

As expected, neurons in the SC deep layers (Fig. $1 A a, B a$ ) and the STN (Fig. $1 A c, B c$ ) failed to respond to the light flash during a baseline period before the SC injection of bicuculline. However, after local injections of bicuculline, both medial and lateral parts of the SC deep layers exhibited short-latency, short-duration bursts of impulses in response to the light flash (Fig. $1 A b, B b$ ). In contrast, a corresponding burst of spikes was recorded from STN neurons only when bicuculline was injected in lateral parts of the SC deep layers (Fig. 1Ad). This effect was observed in all cases $(13 / 13)$. Corresponding injections of bicuculline into medial SC were, however, consistently ineffective (6/6), leaving STN neurons unresponsive to the light flash (Fig. $1 B d$ ). Systematic variations in the STN recording sites cannot account for these differences because they were similarly dispersed throughout the structure for cases receiving medial or lateral SC injections (supplemental Fig. 1, available at www.jneurosci.org as supplemental material). As the visual sensitivity of the $\mathrm{SC}$ was reinstated, several patterns of visually evoked activity emerged in the STN: (1) a short-latency, short-duration phasic response (Fig. 2a); (2) a short-latency response with a slower build up and a longer duration (Fig. $2 b$ ); (3) a superimposition of response types 1 and 2

\section{pre-bicuculline post-bicuculline}

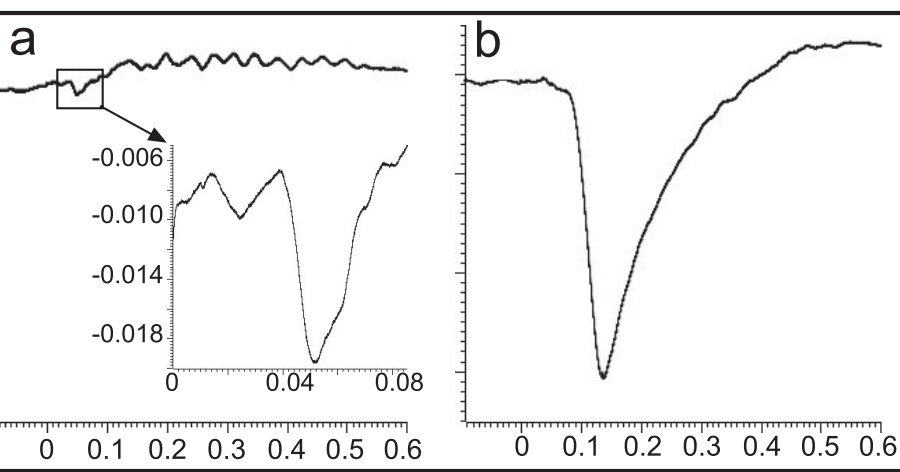

$\begin{array}{lllllll}0 & 0.1 & 0.2 & 0.3 & 0.4 & 0.5 & 0.6\end{array}$

Figure 3. Local application of bicuculline disinhibited visually evoked potentials (VEPs) in visual cortex (compare $\boldsymbol{a}$ and $\boldsymbol{b}$, $\begin{array}{llllll}0 & 0.1 & 0.2 & 0.3 & 0.4 & 0.5\end{array}$

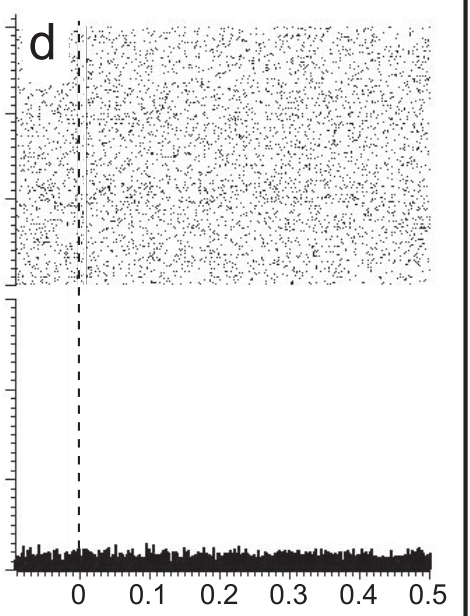

time (s) noting the change of scale in the inset in $\boldsymbol{a}$ ) but failed to restore visual sensitivity to the subthalamic nucleus ( $c$ and $\boldsymbol{d}$ ).

(Fig. 2c); and (4) a long-latency, long-duration response (Fig. $2 d)$. No reliable relationships were established between the various response patterns and either the loci of recording sites in the STN or the precise location of bicuculline injections into the SC (supplemental Fig. 1, available at www.jneurosci.org as supplemental material). However, in all cases the STN visual response latencies (mean latency: $86 \pm 9 \mathrm{~ms}$ ) were reliably longer than those recorded in the SC (mean latency: $53 \pm 5 \mathrm{~ms})(t=3.679$; $\mathrm{df}=12 ; p<0.01)$. Considered together, these results suggest that the lateral SC is an important source of short-latency visual input to the STN.

\section{Effect of SC disinhibition on spontaneous activity in STN}

General disinhibition of neural activity within the SC also induced a significant increase of the tonic firing rate of STN neurons measured in the $500 \mathrm{~ms}$ before each light flash. Thus, the mean prestimulus spike rate of STN multiunit activity increased from $26.62 \pm 2.4 \mathrm{~Hz}$ to $45.17 \pm 3.86 \mathrm{~Hz}$ following injections of bicuculline into the lateral SC $(t=-4.19 ; \mathrm{df}=12 ; p<0.01)$. In contrast, no comparable changes were found in the baseline firing rate of STN neurons following corresponding injections into the medial SC (mean number of multiunit spikes recorded before and after bicuculline were $27.24 \pm 1.7 \mathrm{~Hz}$ and $35.00 \pm 2.6 \mathrm{~Hz}$, respectively; $t=-2.3 ; \mathrm{df}=5 ; p>0.05)$. These data provide further support for a regionally specific functional link between the lateral SC deep layers and the STN. 


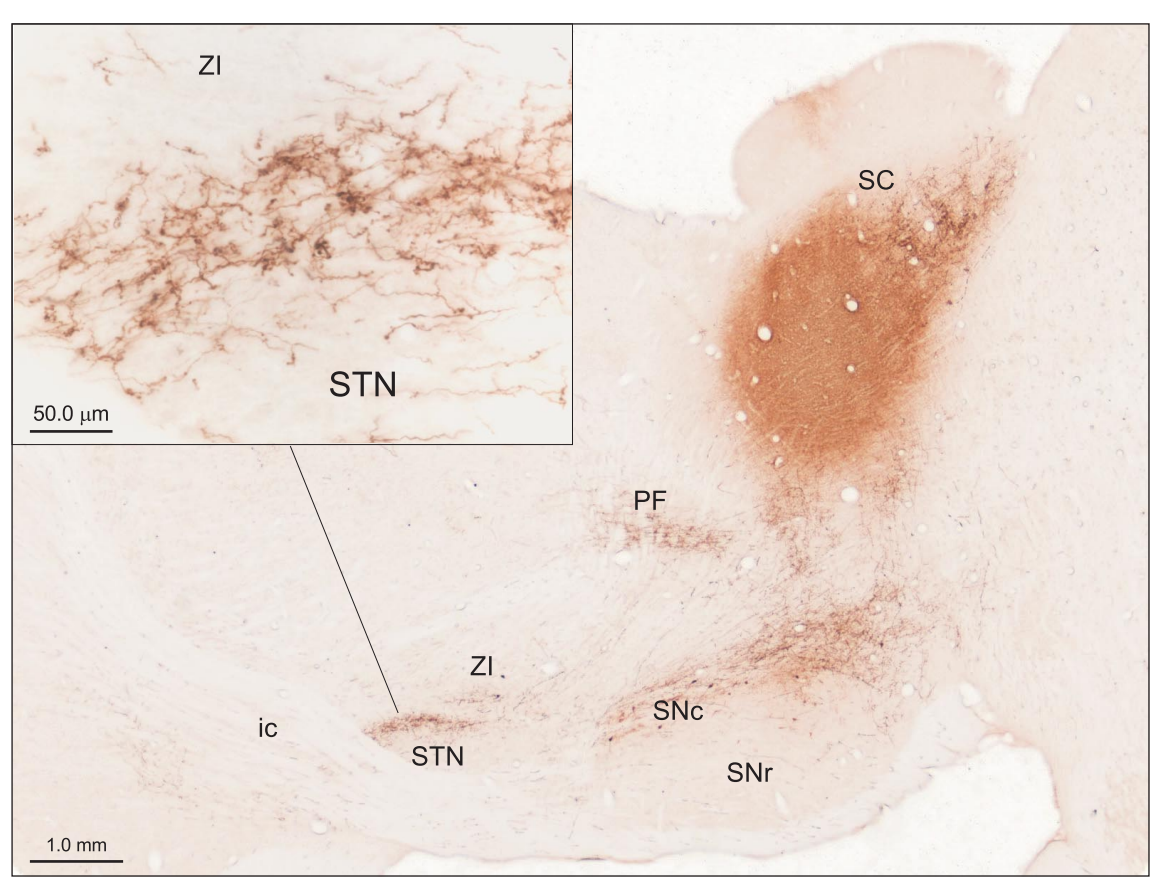

Figure 4. A robust tectosubthalamic projection was evident. Photomicrograph of a parasagittal section of rat brain illustrating the location of a large injection of the anterograde tracer PHA-L in the deep layers of the superior colliculus. Dense labeling of fibers and terminal boutons can be seen in dorsal and rostral STN (see inset) as well as in the parafascicular nucleus of the thalamus (PF) and substantia nigra pars compacta (SNc). ic, Internal capsule; SNr, substantia nigra pars reticulata; ZI, zona incerta.

\section{Disinhibition of visual cortex}

To determine whether enhanced activity in the visual cortex might also influence STN visual activity, bicuculline was applied to the primary visual cortex to increase its responsiveness to visual stimuli. Although the application of bicuculline significantly potentiated local cortical visually evoked activity (Fig. $3 a, b)$, in all cases $(3 / 3)$, the population response of STN neurons remained insensitive to the light flash (Fig. $3 c, d$ ). Once again, the STN recording sites were among those from which clear visual responses had been recorded following disinhibition of the lateral SC (supplemental Fig. 1b, available at www.jneurosci.org as supplemental material). Local disinhibition of the visual cortex also failed to influence the baseline firing rate of STN neurons (mean multiunit spikes recorded before and after bicuculline $26.16 \pm$ $2.85 \mathrm{~Hz}$ and $30.77 \pm 2.79 \mathrm{~Hz}$, respectively; $t=-0.925 ; \mathrm{df}=2$; $p>0.05)$. These data indicate that comparable disinhibition of cortical visual activity was unable to influence either the baseline activity or restore visual sensitivity to STN neurons in the anesthetized preparation.

Together, the electrophysiological data suggest that the presence of short-latency visually evoked activity in the STN is critically dependent on input from the lateral SC deep layers but not from the medial SC or visual cortex. This degree of functional segregation within the SC was at odds with current descriptions of direct connections between the SC and the STN in rodents (Tokuno et al., 1994); a projection that seems, at best, modest with no evidence of regional specialization within the SC. Therefore, to better understand the anatomical basis of our electrophysiological data, we reevaluated the SC-STN pathway in greater detail.

\section{Anatomy}

Anterograde tract tracing: light microscopy

Injections of the anterograde tracers PHA-L $(n=4)$ and/or BDA $(n=8)$ into the SC revealed a more robust direct pathway to the
STN than was previously reported (Tokuno et al., 1994) (Fig. 4). At least two different trajectories for SC efferent fibers could be identified. First, a bundle of fibers was seen leaving the rostral aspect of the $\mathrm{SC}$, coursing vertically down through the parafascicular nucleus and then turning rostrally along the medial lemniscus to terminate in the STN. The second route appeared as a rostral extension of the pathway we have previously described (Comoli et al., 2003) from the SC to dopaminergic neurons in the ventral midbrain, the tectonigral pathway (Fig. 4).

The tectosubthalamic projection originates almost exclusively in the lateral SC. Thus, injections of either PHA-L or BDA into lateral regions $(n=6)$ were associated with high densities of labeled axons and terminals in ipsilateral STN (Figs. 4, 5a-c). In contrast, the STN was virtually devoid of terminal labeling when injections $(n=$ 6) were directed to the medial SC (Fig. $5 d-$ f). Moreover, tectosubthalamic axons and terminals were differentially distributed within specific subregions of the STN. In the caudal aspects of the STN, anterogradely labeled axons and terminals were largely confined in a dorsal sheet (Fig. 4) that extended across the entire mediolateral dimension of the nucleus (Fig. 5c). Rostrally, terminating fibers filled the entire mediolateral and dorsoventral area of the STN (Fig. 5b). There were many instances where anterogradely labeled boutons were seen in close apposition to STN soma and dendrites (Fig. $5 g-j$ ).

\section{Anterograde tract tracing: electron microscopy}

To determine whether the appositions between terminals labeled from the SC and neuronal elements in the STN established direct synaptic contacts, an electron microscopic analysis was performed on tissue from three rats that received PHA-L injections into the lateral SC. A total of 85 labeled structures were examined, of which 27 were identified as synaptic boutons. These were found to form synaptic contacts with 32 postsynaptic elements within the STN. In each case, the synapse was of the asymmetrical type (Gray's type 1). The labeled terminals made synaptic contact either with dendritic shafts $(53 \%, 17 / 32)$ (Fig. $6 a)$ or spines $(47 \%$, 15/32) (Fig. 6b) of STN neurons, with a small population of boutons making contact with both $(19 \%, 5 / 27)$. These results demonstrate that there is a direct synaptic connection between the SC and the STN and the type of synapse formed suggests that the projection is predominantly excitatory.

\section{Retrograde tract tracing: light microscopy}

To identify the regional distribution and morphology of tectosubthalamic projection neurons, labeled neurons in the SC were plotted following injections of retrograde tracers $\mathrm{CTb}(n=3)$ or True Blue $(n=3)$ into the STN. Retrogradely labeled neurons were mainly located in lateral sectors of the SC deep layers (Fig. 7). This impression was confirmed by a three-factor (layers-five levels; medial/lateral—two levels; rostral/caudal-four levels) repeated-measures ANOVA (interaction between the layers and medial/lateral factors: $F=5.254, \mathrm{df}=3, p<0.05)$. Collicular neurons retrogradely labeled by STN injections typically were 


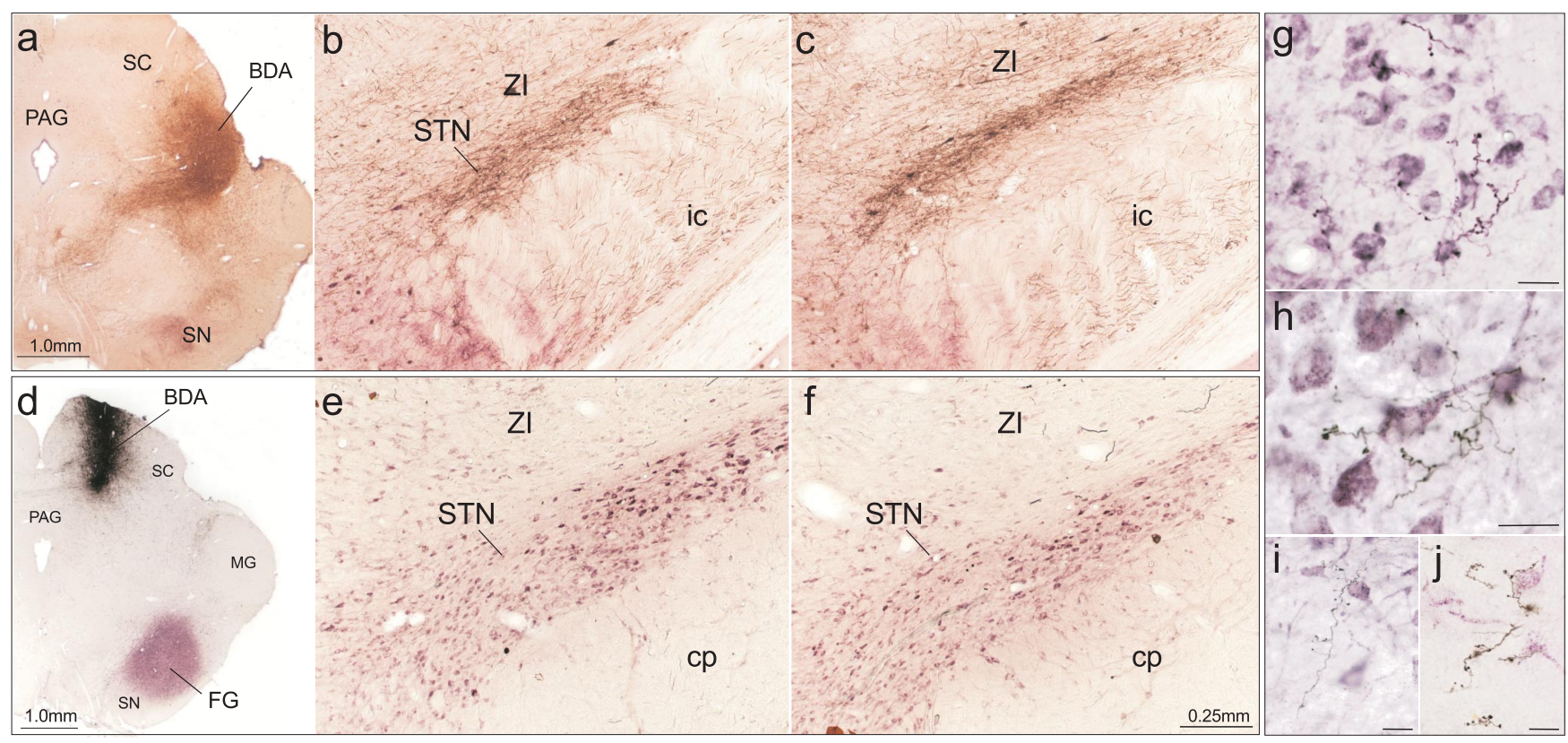

Figure 5. The tectosubthalamic pathway is regionally specific. A coronal section illustrating a biotinylated dextran amine (BDA; anterograde tracer) injection site in the lateral deep layers of the SC (a), associated with dense labeling of terminals in the rostral (b) and central (c) STN. A corresponding injection of BDA into the medial SC (d) produced virtually no anterograde labeling at the same rostrocaudal levels of the STN $(\boldsymbol{e}, \boldsymbol{f})$. In the latter case, neurons of the STN marked with purple dye were retrogradely labeled by an injection of Fluorogold in substantia nigra (SN) and reacted with Vector VIP chromogen $(\boldsymbol{d}) \cdot \boldsymbol{g}-\boldsymbol{j}$, Examples of terminals and boutons anterogradely labeled from the lateral SC in close apposition with STN neurons retrogradely labeled from SN. Scale bar in $\boldsymbol{f a p p l i e s}$ to $\boldsymbol{b}-\boldsymbol{d}$, whereas scale bars in photomicrographs $\boldsymbol{g}-\boldsymbol{j}=25 \mu \mathrm{m}$. cp, Cerebral peduncle; MG, medial geniculate nucleus; PAG, periaqueductal gray; Zl, zona incerta.

small-to-medium-sized multipolar neurons (Saito and Isa, 1999) (mean cell-body area $=182.5 \mu \mathrm{m}^{2} \pm 12.7 \mathrm{SE}$; range $85.6-$ $417.8 \mu \mathrm{m}^{2)}$ (Fig. 8). Large tectoreticulospinal output neurons (Bickford and Hall, 1989) were not labeled.

In summary, our tracing experiments demonstrated a substantial anatomical projection between the SC and the STN that originates from small-to-mediumsized neurons concentrated in the far lateral aspects of the deep SC layers. Tectosubthalamic fibers preferentially target dorsal and rostral parts of the STN where they make asymmetric, presumed excitatory, synaptic contacts.

\section{Discussion}

The main findings of the present study are as follows: (1) local pharmacological disinhibition of the lateral, but not medial SC deep layers, nor comparable disinhibition of the visual cortex, leads to a short-latency visual activation of the STN; (2) the direct projection from the SC to the STN is more substantial than previously proposed (Tokuno et al., 1994), arising predominantly from the lateral SC deep layers and terminating preferentially in the rostral and dorsal sectors of the STN; and (3) both electrophysiological and electron-microscopic data suggest that the pathway is predominantly excitatory. Together, these results identify the lateral SC as the principal, if not exclusive, source of short-latency visual input to the STN. These findings have broad implications for understanding the functional role(s) of the STN within the wider context of the basal ganglia (see below).

We have demonstrated that STN neurons respond at short latency to visual stimuli and that this response derives from the SC. A close association was observed between the location within the SC where local pharmacological disinhibition was required for visual stimuli to elicit short-latency activation of the STN and tectosubthalamic cells of origin. Thus, the tectosubthalamic projection arises mainly from small-to-medium-sized neurons in the lateral deep layers of the SC, where pharmacological disinhibition was effective. Virtually no anterogradely labeled fibers and/or terminals were observed in the STN following tracer injections into the medial SC deep layers and bicuculline injections into the medial SC left the STN visually unresponsive, despite the presence of robust visually evoked population responses from medial deep SC layer neurons.

A second point of correspondence between the electrophysiological and anatomical data concerns the potential excitatory nature of the tectosubthalamic projection. Unlike our previous electron-microscopic analysis of the projection from the SC to dopaminergic neurons in the ventral midbrain (the tectonigral projection), where both asymmetric (presumed excitatory) and symmetric (presumed inhibitory) synaptic contacts were reported (Comoli et al., 2003), only labeled terminals forming asymmetric synapses were seen in the present study. Consistent with these anatomical findings, electrophysiological analysis of the tectonigral projection (Dommett et al., 2005) revealed both 

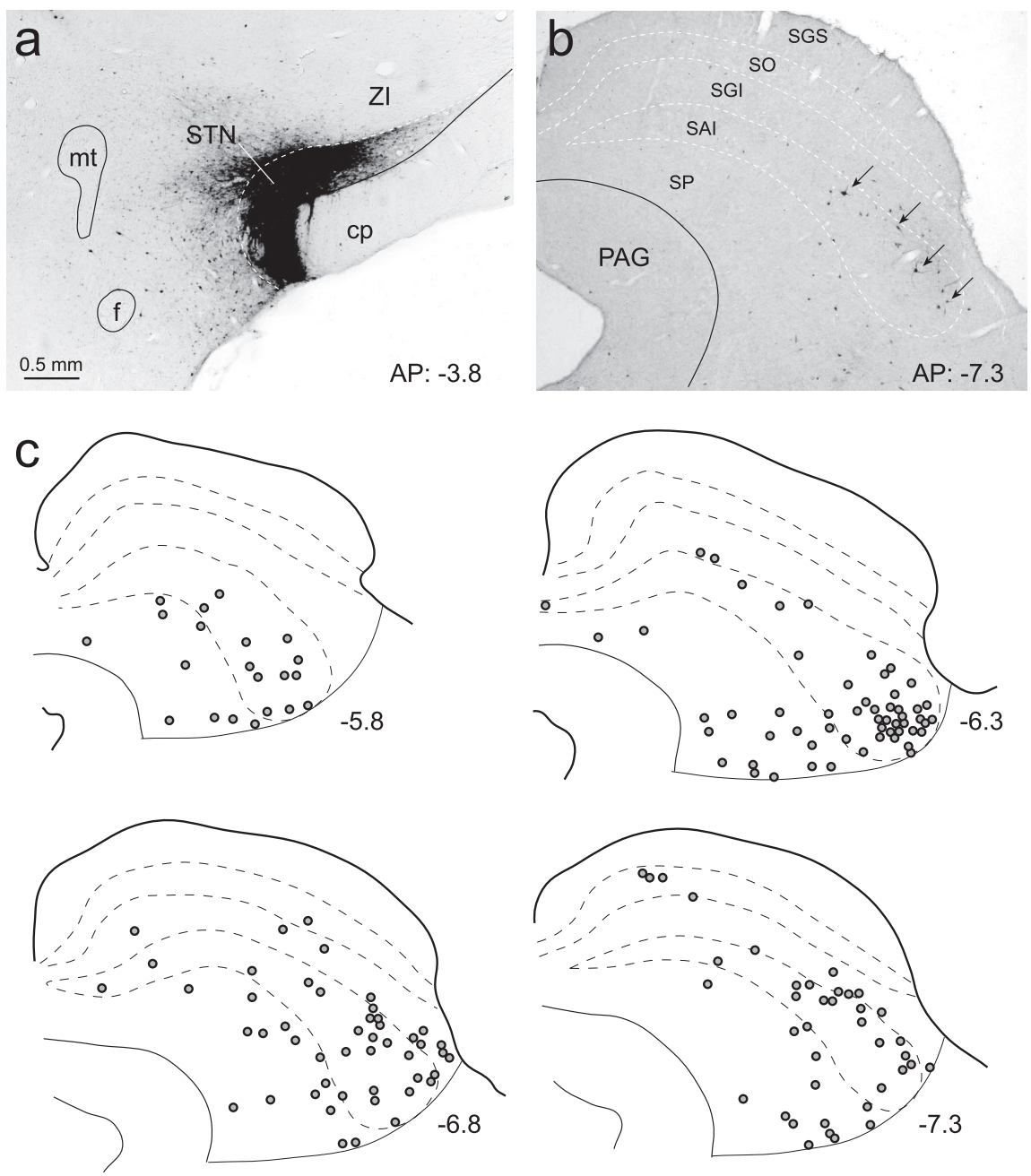

Figure 7. Collicular neurons retrogradely labeled from the STN are concentrated in the lateral deep layers. $\boldsymbol{a}$, A photomicrograph of a CTb (retrograde tracer) the injection site confined to the STN. $\boldsymbol{b}$, A photomicrograph of retrogradely labeled neurons (arrows) concentrated in the deep layers of the SC. c, A quantitative plot of labeled neurons at different anterior-posterior levels of the SC (numbers associated with each section indicates its location caudal to bregma in millimeters). cp, Cerebral peduncle; $f$, fornix; mt, mammillothalamic tract; PAG, periaqueductal gray; ZI, zona incerta.

excitatory and inhibitory visually evoked responses in nigral dopaminergic neurons. No inhibitory responses to visual stimulation were observed in the present study. However, some slight caution is required because inhibitory responses of individual STN neurons may have been masked by the dominating excitatory responses of others in the multiunit record. Despite this qualification, the fact that we observed only excitatory visually evoked multiunit responses in the STN is consistent with our failure to detect symmetrical tectosubthalamic synaptic contacts. To this extent, it appears that the tectonigral and tectosubthalamic projections may be at least partly anatomically and functionally distinct.

An interesting observation in the present study was that, while visually evoked responses could be recorded from locations throughout the STN, our anterograde tracing data showed that injections of tracer into the lateral SC consistently produced dense terminal labeling confined to a specific region of the STN (rostral and dorsal). There could be several reasons for this apparent inconsistency. First, in the rat, STN neurons typically have large dendritic arbors that can extend over half the length of the nucleus (Kita et al., 1983; Afsharpour, 1985a). In addition, many of these glutamatergic STN projection neurons (Smith et al.,
1998) have intranuclear (local) axon collaterals making intrinsic, presumed excitatory connections within the STN (Chang et al., 1983). Neural modeling of this intrinsic architecture suggests that large regions of this highly interconnected nucleus respond to excitatory input with a widespread pulse of increased activity (Gillies and Willshaw, 1998). If these biologically constrained simulations accurately reflect STN neural processing, excitatory visual input directed to one region of the STN (e.g., rostral and dorsal regions) could evoke responses throughout the structure. However, the extent to which intrinsic STN neurons are effectively interconnected has been questioned (Wilson et al., 2004), and following electrical stimulation of different regions of cerebral cortex, spatially restricted zones of excitation were found within the STN (Kolomiets et al., 2001). A second possible explanation is that the observation of qualitatively different patterns of visually evoked responses from the STN suggests the involvement of additional afferent pathways. In the light of similar findings following electrical stimulation of cerebral cortex, Kolomiets et al. (2001) concluded that different classes of electrophysiological response pattern in the STN probably reflected neural transmission along the different direct and indirect routes connecting the cerebral cortex with the STN (Maurice et al., 1998; Nambu et al., 2002). A similar explanation could account for the different classes of visually evoked responses recorded throughout the STN in the present study. Possible indirect routes might include relays in the parafascicular nucleus of the thalamus (Maurice et al., 1998; Krout et al., 2001), the pedunculopontine tegmental nucleus (Redgrave et al., 1987; Spann and Grofova, 1989), and dopaminergic neurons of substantia nigra (Tofighy et al., 2003; Dommett et al., 2005). The potential role of these indirect projections and the extent to which they may express divergent contacts remains to be determined.

\section{Functional implications}

Both the SC and the basal ganglia are phylogenetically ancient systems that have been highly conserved throughout vertebrate evolution (Medina and Reiner, 1995). Interactions between the SC and the basal ganglia would, therefore, have been established before the elaboration of cerebral cortex. Indeed, we have argued previously that, while closed-loop projections are an important component of corticobasal ganglia-cortical architecture (Alexander et al., 1986; Parent and Hazrati, 1995), a similar, but phylogenetically older, closed-loop architecture involving the striatum connects the basal ganglia with brainstem sensorimotor structures, including the SC (McHaffie et al., 2005). In recent years, it has been appreciated that direct connections between the cerebral cortex and the STN, the hyperdirect pathway (Nambu et al., 2002), represents a second important route providing input to 


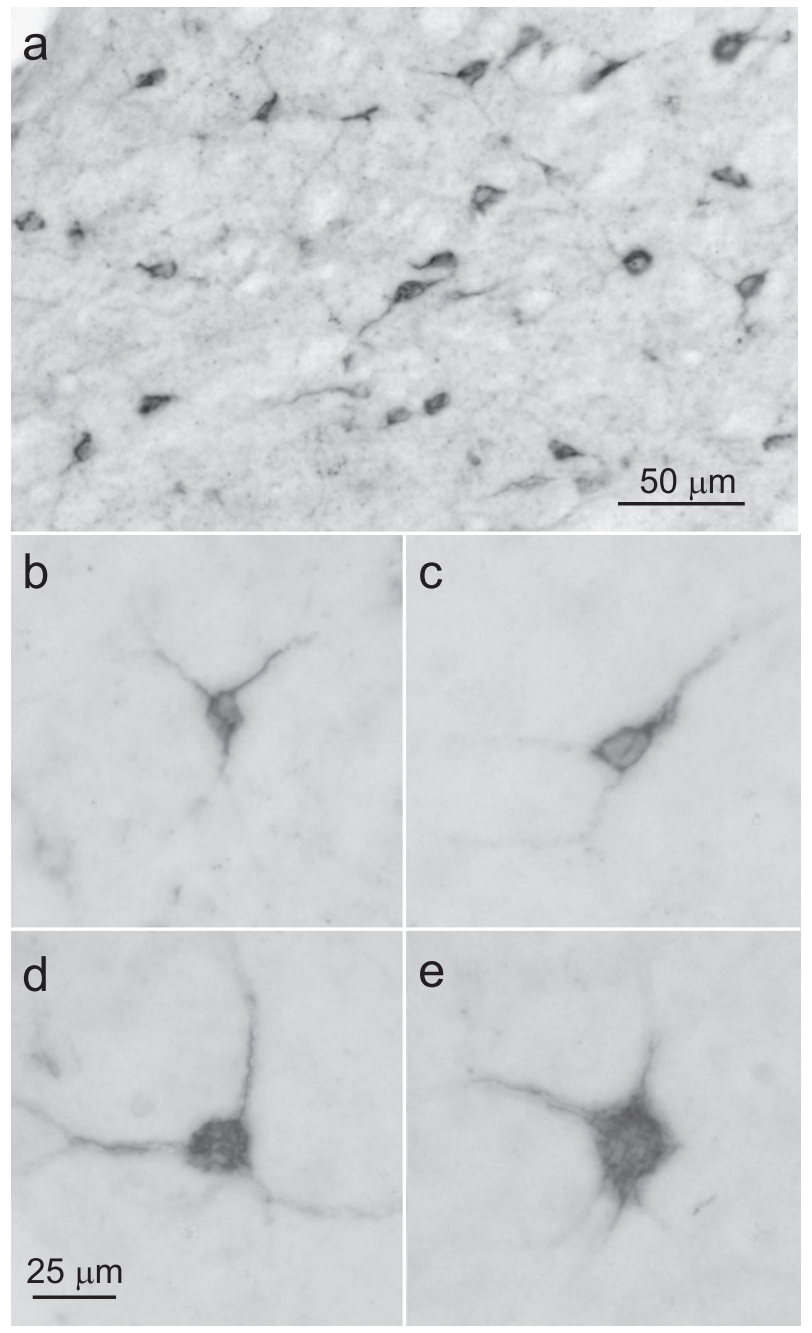

Figure 8. Morphology of retrogradely labeled collicular neurons following an injection of CTb into the STN. $\boldsymbol{a}$, A concentration of small ( $\boldsymbol{b}$ and $\boldsymbol{c}$ ), with a few medium-sized ( $\boldsymbol{d}$ and $\boldsymbol{e}$ ), multipolar neurons was located in the lateral deep layers of the SC. Scale bar in $\boldsymbol{d}$ also applies to $b, c$, and $e$.

the basal ganglia. The present demonstration of a significant projection to the STN from an evolutionarily primitive sensorimotor structure (i.e., the SC) reveals a further architectural correspondence between cortical and subcortical connections with the basal ganglia.

Clues to the function(s) of the STN have been gleaned by examining its impact on basal ganglia target structures such as the SC. The STN provides a major input to the basal ganglia output nuclei, including the substantia nigra pars reticulata, which contains the cells of origin of the nigrotectal projection to the SC. Because the subthalamonigral projection is excitatory (Smith et al., 1998) and the nigrotectal pathway is inhibitory (Chevalier and Deniau, 1990), a burst of excitatory STN activity transmitted to nigrotectal neurons would deliver a burst of inhibitory activity to the SC: similar inhibitory signals would appear simultaneously at other projection targets of the basal ganglia output nuclei. Two potential, not necessarily exclusive, functions for such inhibition have been proposed: (1) Since the SC plays an important role in directing head and eye movements (i.e., gaze shifts) toward or away from sudden changes in the visual field (Wurtz and Albano, 1980), a short-latency burst of inhibitory activity could be part of a mechanism to interrupt current gaze fixation before the direc- tion of gaze (Gillies and Willshaw, 1998; Redgrave et al., 1999). Inhibitory signals directed elsewhere could interrupt other components of behavioral output. (2) A phasic, widely broadcast excitatory signal from the STN to basal ganglia output nuclei could provide the prior "excitatory surround" on which later focused inhibition from the striatum (representing the desired motor program for execution) is superimposed during action selection, in which the basal ganglia are believed to play a role (Mink, 1996; Gurney et al., 2001). The findings that visual responses in STN neurons have shorter latencies than those of gaze shifts required to fixate a target (i.e., STN latencies are generally $<100 \mathrm{~ms}$ compared with normal saccadic latencies of $>130 \mathrm{~ms}$ in the monkey; Schiller et al., 2008), and that short-latency input is provided by the SC, whose visual processing capabilities are rapid but relatively limited (Wurtz and Albano, 1980; Stein and Meredith, 1993), are consistent with both of the proposed putative functions, which require afferent sensory signals to the STN to be rapid but care less about the detailed content of those signals.

However, additional results in the present study raise problems for both suggestions. The ecology of the rodent is such that threatening stimuli (i.e., predators), presumably requiring the interruption of behavior, are most commonly detected in the upper visual field, which according to the retinocentric map is represented in the medial SC (Dean et al., 1989). Yet, the present study revealed virtually no contribution from the medial SC to the tectosubthalamic projection, and hence no clear means for upper field stimuli to interrupt behavior via this route. On the other hand, from an action selection point of view, it is not immediately apparent why selection mechanisms concerned with responses to visual events in the upper field require a radically different, non-STN-associated architecture, compared with that used for the lower field. At the very least, the present results are inconsistent with the STN playing the global, ubiquitous role in action selection/behavioral interruption envisaged, although at a deeper level they may suggest that a reappraisal of the function of this nucleus is required.

\section{References}

Afsharpour S (1985a) Light microscopic analysis of Golgi-impregnated rat subthalamic neurons. J Comp Neurol 236:1-13.

Afsharpour S (1985b) Topographical projections of the cerebral cortex to the subthalamic nucleus. J Comp Neurol 236:14-28.

Albers FJ, Meek J, Nieuwenhuys R (1988) Morphometric parameters of the superior colliculus of albino and pigmented rats. J Comp Neurol 274:357-370.

Alexander GE, DeLong MR, Strick PL (1986) Parallel organization of functionally segregated circuits linking basal ganglia and cortex. Annu Rev Neurosci 9:357-381.

Benabid AL (2003) Deep brain stimulation for Parkinson's disease. Curr Opin Neurobiol 13:696-706.

Bickford ME, Hall WC (1989) Collateral projections of predorsal bundle cells of the superior colliculus in the rat. J Comp Neurol 283:86-106.

Chang HT, Kita H, Kitai ST (1983) The fine structure of the rat subthalamic nucleus: an electron microscopic study. J Comp Neurol 221:113-123.

Chevalier G, Deniau JM (1990) Disinhibition as a basic process in the expression of striatal functions. Trends Neurosci 13:277-280.

Comoli E, Coizet V, Boyes J, Bolam JP, Canteras NS, Quirk RH, Overton PG, Redgrave P (2003) A direct projection from superior colliculus to substantia nigra for detecting salient visual events. Nat Neurosci 6:974-980.

Dean P, Redgrave P, Westby GWM (1989) Event or emergency? Two response systems in the mammalian superior colliculus. Trends Neurosci 12:137-147.

DeLong MR, Crutcher MD, Georgopoulos AP (1985) Primate globus pallidus and subthalamic nucleus: functional organization. J Neurophysiol 53:530-543.

Dommett E, Coizet V, Blaha CD, Martindale J, Lefebvre V, Walton N, May- 
hew JE, Overton PG, Redgrave P (2005) How visual stimuli activate dopaminergic neurons at short latency. Science 307:1476-1479.

Gerfen CR, Wilson CJ (1996) The basal ganglia. In: Handbook of chemical neuroanatomy, Vol 12: Integrated systems of the CNS, Part III. (Swanson LW, Bjorklund A, Hokfelt T, eds), pp 371-468. Amsterdam: Elsevier.

Gillies AJ, Willshaw DJ (1998) A massively connected subthalamic nucleus leads to the generation of widespread pulses. Proc Biol Sci 265:2101-2109.

Gurney K, Prescott TJ, Redgrave P (2001) A computational model of action selection in the basal ganglia. II. Analysis and simulation of behaviour. Biol Cybern 84:411-423.

Joel D, Weiner I (1997) The connections of the primate subthalamic nucleus: Indirect pathways and the open-interconnected scheme of basal ganglia-thalamocortical circuitry. Brain Res Rev 23:62-78.

Katsuta H, Isa T (2003) Release from GABA(A) receptor-mediated inhibition unmasks interlaminar connection within superior colliculus in anesthetized adult rats. Neurosci Res 46:73-83.

Kita H, Chang HT, Kitai ST (1983) The morphology of intracellularly labeled rat subthalamic neurons: a light microscopic analysis. J Comp Neurol 215:245-257.

Kolomiets BP, Deniau JM, Mailly P, Ménétrey A, Glowinski J, Thierry AM (2001) Segregation and convergence of information flow through the cortico-subthalamic pathways. J Neurosci 21:5764-5772.

Krout KE, Loewy AD, Westby GWM, Redgrave P (2001) Superior colliculus projections to midline and intralaminar thalamic nuclei of the rat. J Comp Neurol 431:198-216.

Matsumura M, Kojima J, Gardiner TW, Hikosaka O (1992) Visual and oculomotor functions of monkey subthalamic nucleus. J Neurophysiol 67:1615-1632.

Maurice N, Deniau JM, Glowinski J, Thierry AM (1998) Relationships between the prefrontal cortex and the basal ganglia in the rat: physiology of the corticosubthalamic circuits. J Neurosci 18:9539-9546.

McHaffie JG, Stanford TR, Stein BE, Coizet V, Redgrave P (2005) Subcortical loops through the basal ganglia. Trends Neurosci 28:401-407.

Medina L, Reiner A (1995) Neurotransmitter organization and connectivity of the basal ganglia in vertebrates: implications for evolution of basal ganglia. Brain Behav Evol 46:235-258.

Mink JW (1996) The basal ganglia: focused selection and inhibition of competing motor programs. Prog Neurobiol 50:381-425.

Nambu A, Tokuno H, Takada M (2002) Functional significance of the cortico-subthalamo-pallidal 'hyperdirect' pathway. Neurosci Res 43:111-117.

Obeso JA, Rodríguez-Oroz MC, Rodríguez M, Lanciego JL, Artieda J, Gonzalo N, Olanow CW (2000) Pathophysiology of the basal ganglia in Parkinson's disease. Trends Neurosci 23:S8-S19.

Parent A, Hazrati LN (1995) Functional anatomy of the basal ganglia. 2. The place of subthalamic nucleus and external pallidum in basal ganglia circuitry. Brain Res Rev 20:128-154.
Paxinos G, Watson C (1997) The rat brain in stereotaxic coordinates. Sydney: Academic.

Redgrave P (2007) Basal ganglia. Scholarpedia 2:1825.

Redgrave P, Mitchell IJ, Dean P (1987) Descending projections from the superior colliculus in rat: a study using orthograde transport of wheatgerm-agglutinin conjugated horseradish peroxidase. Exp Brain Res 68:147-167.

Redgrave P, Marrow L, Dean P (1992) Topographical organization of the nigrotectal projection in rat: evidence for segregated channels. Neuroscience 50:571-595.

Redgrave P, Prescott TJ, Gurney K (1999) The basal ganglia: a vertebrate solution to the selection problem? Neuroscience 89:1009-1023.

Sahibzada N, Dean P, Redgrave P (1986) Movements resembling orientation or avoidance elicited by electrical stimulation of the superior colliculus in rats. J Neurosci 6:723-733.

Saito Y, Isa T (1999) Electrophysiological and morphological properties of neurons in the rat superior colliculus. I. Neurons in the intermediate layer. J Neurophysiol 82:754-767.

Schiller PH, Kendall GL, Slocum WM, Tehovnik EJ (2008) Conditions that alter saccadic eye movement latencies and affect target choice to visual stimuli and to electrical stimulation of area V1 in the monkey. Vis Neurosci 25:661-673.

Smith Y, Bevan MD, Shink E, Bolam JP (1998) Microcircuitry of the direct and indirect pathways of the basal ganglia. Neuroscience 86:353-387.

Spann BM, Grofova I (1989) Origin of ascending and spinal pathways from the nucleus tegmenti pedunculo-pontinus in the rat. J Comp Neurol 283:13-27.

Stein BE, Meredith MA (1993) The merging of the senses. Cambridge, MA: MIT.

Telford S, Wang S, Redgrave P (1996) Analysis of nociceptive neurones in the rat superior colliculus using c-fos immunohistochemistry. J Comp Neurol 375:601-617.

Tofighy A, Abbott A, Centonze D, Cooper AJ, Noor E, Pearce SM, Puntis M, Stanford IM, Wigmore MA, Lacey MG (2003) Excitation by dopamine of rat subthalamic nucleus neurones in vitro- a direct action with unconventional pharmacology. Neuroscience 116:157-166.

Tokuno H, Takada M, Ikai Y, Mizuno N (1994) Direct projections from the deep layers of the superior colliculus to the subthalamic nucleus in the rat. Brain Res 639:156-160.

Wichmann T, DeLong MR (1996) Functional and pathophysiological models of the basal ganglia. Curr Opin Neurobiol 6:751-758.

Wilson CL, Puntis M, Lacey MG (2004) Overwhelmingly asynchronous firing of rat subthalamic nucleus neurones in brain slices provides little evidence for intrinsic interconnectivity. Neuroscience 123:187-200.

Wurtz RH, Albano JE (1980) Visual-motor function of the primate superior colliculus. Annu Rev Neurosci 3:189-226. 